\title{
CHROMOIONOPHORES IN OPTICAL ION SENSORS
}

\author{
J. VAN GENT ${ }^{a}, b$, P. V. LAMBECK ${ }^{a}$, H. J. M. KREUWEL ${ }^{a}$, G. J. GERRITSMA ${ }^{a}$, \\ E. J. R. SUDHÖLTER ${ }^{b}$, D. N. REINHOUDT ${ }^{b}$ and Th. J. A. POPMA ${ }^{a}$ \\ ${ }^{a}$ Laboratory of Material Sciences, ${ }^{b}$ Laboratory of Organic Chemistry, University of \\ Twente, P.O. Box 217, 7500 AE Enschede (The Netherlands)
}

\section{Abstract}

The feasibility of surface plasmon resonance (SPR) for ion sensing has been investigated. Emphasis is laid on a simulation-based optimization of the SP carrying structure, as well as the applicability of a specific chemo-optical interface we have developed. A preliminary result is presented.

\section{Introduction}

The central part of a solid-state chemo-optical transducer is formed by a material that is able to transform a variation of the concentration of a specific chemical species in its environment into a variation of its dielectric function, namely the chemo-optical interface layer. This change in dielectric properties may arise from a wide variety of chemical or physical interactions. Complexation of molecules of the sensing material with those of the chemical species is one of the most promising concepts, because this complexation can be made selective and reversible by choosing appropriate receptor molecules. A fast response time can be realized by applying these materials in the form of very thin layers, preferably monolayers. The change in dielectric function of these thin interface layers can be excellently probed by the surface plasmon resonance (SPR) technique, as was first pointed out by Liedberg et al. [1]. By coupling the surface plasmon supporting structure to a planar waveguiding system, Kreuwel et al. [2] developed an SPR-based solid-state microsensor, thus abandoning the rather bulky optical equipment commonly used. For practical applications, the required sensor performance has to be specified as to selectivity, measurement range, sensitivity and response time. Special chemo-optical interface layers have to be developed to meet these requirements. In this development a central position has to be given to the engineering of the molecules responsible for the chemo-optical transduction.

Chromoionophores [3] (i.e., molecules changing their colour on complexation with the compound that is to be measured) are very promising for producing large chemo-optical transduction effects. Appropriate methods of immobilizing these chromoionophores at the interface have to be developed. 
In this paper we will focus our attention on the optimization of a chromoionophoric layer for use in SPR sensors; also some aspects of the performance of an SPR sensor provided with this layer will be discussed.

In Section 1 the main features of the surface-plasmon resonance technique are described and in Section 2 we present the simulation-based, optimal surface-plasmon supporting structure. In Section 3 we concentrate on the chromoionophores. Attention is paid to the immobilization of these molecules in Section 4 and some aspects of sensor performance are given and discussed in Section 5.

\section{SPR technique}

A surface plasmon is an electromagnetic wave propagating along the surface of a material showing a negative dielectric constant (e.g., silver). In an SPR sensor the chemo-optical interface layer is usually attached directly to the metal, as shown in Fig. 1 , where the metal surface corresponds to the $x=0$ plane.

The electric field $E$ of the surface plasmon propagating in the $z$-direction can be calculated straightforwardly from Maxwell's equations [4] and is approximated as:

$\tilde{E}_{x}^{0}(x, z, t)={\tilde{E_{x}}}^{0}(x) \operatorname{exp~i}\left(\omega t-\tilde{k}_{z} \cdot z\right)$

where

$\omega$ = angular frequency

and

$\tilde{k}_{z}=k_{z}{ }^{\prime}+\mathrm{i} \cdot \boldsymbol{k}_{z}{ }^{\prime \prime}$

is the propagation constant.

The field amplitude $E_{x}{ }^{0}(x)$ peaks at the interface and decays exponentially in the adjacent half spaces. The decay constants are of the order of $10^{-1}-10^{-3} \mathrm{~nm}^{-1}$, representing the large field concentration in the interface region. The propagation constant of a surface plasmon propagating along the interface of two semi-infinite layers is given by the dispersion relation:

$\tilde{k}_{z}=\frac{\omega}{c}\left(\frac{\widetilde{\epsilon}_{\mathrm{Ag}} \tilde{\epsilon}_{\mathrm{D}}}{\tilde{\epsilon}_{\mathrm{Ag}}+\tilde{\epsilon}_{\mathrm{D}}}\right)^{1 / 2}$

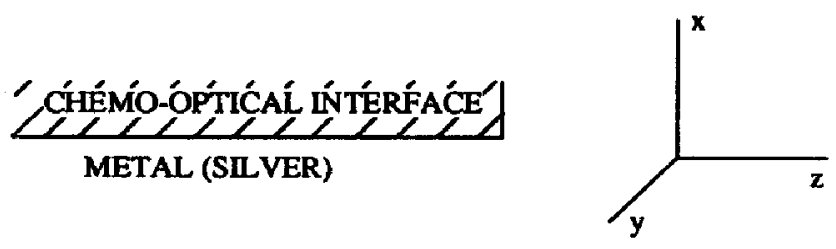

Fig. 1. Surface plasmon resonance at the interface of a metal and a dielectric material. 


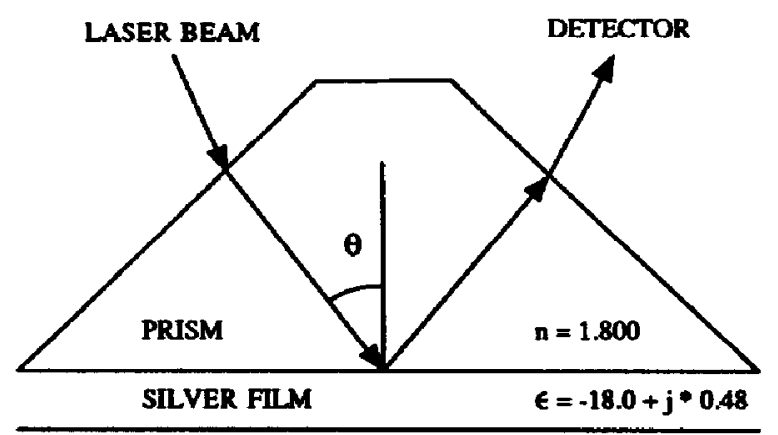

SENSING LAYER (immobilied chromoionophore)

EXTERNAL ENVIRONMENT (AIR OR WATER)

Fig. 2. Experimental set-up for the detection of surface plasmon modes (Kretschmann configuration).

where $c=$ velocity of light, and $\epsilon_{\mathrm{Ag}}, \epsilon_{\mathrm{D}}=$ dielectric constants of the silver and the dielectric layer, respectively.

For non semi-infinite layers, $E_{x}{ }^{0}$ and $k_{z}$ have to be calculated numerically (see Section 2). It is the change in $k_{z}$ caused by the complexation-originated change of $\epsilon_{\mathrm{D}}$ that has to be measured. The commonly used experimental set-up, the Kretschmann configuration [5], is symbolized in Fig. 2.

The surface plasmon supported by the silver sensing-layer interface will be excited only if the condition

$k \sin \theta=k_{z}$

is obeyed, where

$k=2 \pi n_{\mathrm{p}} / \lambda_{\mathrm{o}}$

$n_{\mathrm{p}}$ is the refractive index of the prism and $\lambda_{0}$ is the laser beam wavelength in vacuum.

The surface plasmon resonance is experimentally observed as a very sharp minimum of the light reflectance on variation of the angle of incidence $\theta$. Representative curves are sketched in Fig. 3.

As is common in resonance phenomena, the resonance is spread by damping. In this case the damping originates from the imaginary parts of the dielectric constants of the silver and interface layers, the surface roughness and the coupling of the surface plasmon with the radiation field in the prism. The potential to utilize a surface plasmon for sensing purposes depends on the magnitude of the shift $\Delta k_{z}{ }^{\prime}$ caused by a given change $\Delta \epsilon(\lambda)$ of the chemo-optical interface-layer and on the damping of the resonance $k_{z}{ }^{\prime \prime}$. This can be expressed by a relative figure of merit $S$, defined as

$S=\Delta k_{z}{ }^{\prime} / k_{z}{ }^{\prime \prime}$

$\Delta k_{z}{ }^{\prime}$ and $k_{z}{ }^{\prime \prime}$ can be obtained from the $R(\theta)$ reflectance curves, as they are proportional to the shift of the resonance peak and its half width, respectively. The plasmon structure has to be designed to maximize $S$. 


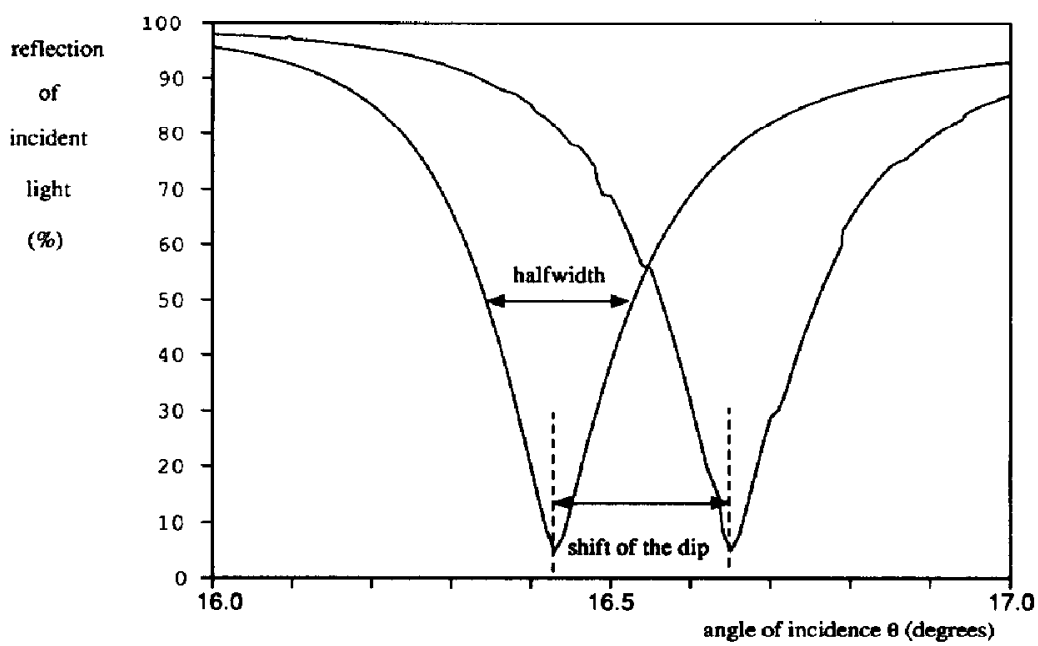

Fig. 3. SPR-reflectance curves. The light reflection is recorded as a function of the angle of incidence $\theta$.

\section{The structure of the SP supporting system}

The structure of the surface plasmon sensor we will discuss is based on the commonly used structure of Fig. 2. However, an intermediate layer of $\mathrm{ZrO}_{2}$ between the silver film and the chemo-optical interface has been added for the following reasons:

(1) Protection of the silver film. The silver surface is very reactive. In particular, sulphur-containing compounds in the environment cause a rapid degradation of the silver film.

(2) Immobilization of the sensing material. The sensing layer usually consists of organic molecules that are more or less soluble in water. The life of the sensor can be greatly extended by an oxidic intermediate layer that is suitable for binding the sensing molecules, thus extending the lifetime of the sensor.

(3) Concentration of the electric field. Applying an intermediate layer with a high refractive index $\left(\mathrm{ZrO}_{2}, n=2.1\right)$ enhances the field in the chemical interface.

The surface plasmon supporting structure has to be designed to optimize the figure of merit $S$. In order to obtain an $S$-value from relation (5) for different SP structures, each characterized by the thickness and complex dielectric constants of the constituting layers, we developed a computer program (SP-DIP [6]) to calculate the reflection curves $R(\theta)$. The imaginary part $\epsilon^{\prime \prime}$ of the dielectric constant $\epsilon$ of the interface layer as a function of the wavelength is easily calculated from absorption spectra. With the aid of our computer program REFRAC, based on Kramers-Kronig relations [7] we are able to calculate the variation of $\epsilon^{\prime}$ with the wavelength from the absorption spectrum of a dye. Using methods like ellipsometry, a reference point has to be determined in order to obtain the absolute level of the curves. 
Another computer program (FIELD [8]) enables us to calculate fieldamplitude distributions in the sensor. In the case of a very thin or weakly absorbing interface, these calculations lead to the following conclusions:

(1) The optimal thickness of the silver layer is $55 \mathrm{~nm}$.

(2) For the intermediate $\mathrm{ZrO}_{2}$ layer, a thickness of about $20 \mathrm{~nm}$ results in maximum $S$ [6]. This thickness is sufficient for protection of the silver film.

\section{Chromoionophores}

We can learn from the computer simulations of the SPR sensor performance that the chromoionophore has to show a strong and narrow absorption band $\left(\epsilon \geqslant 5 \times 10^{4} 1 \mathrm{~mol}^{-1} \mathrm{~cm}^{-1}\right)$ in the visible light region, preferably at a higher wavelength, that will be shifted on complexation over a $\lambda$-distance of at least half the bandwidth. The maximal value of the figure of merit $S$ appears to be obtained at the high wavelength tail of the absorption bands. Complexation has to be selective and immobilization of the chromoionophores in the sensor layer has to be possible. Chromophores showing the required high extinction coefficient, a narrow absorption band and absorption at a high wavelength can be found in the class of the merocyanin dyes. We have synthesized a chromoionophore, consisting of such a merocyanin dye (chromophore) and a crown ether (ionophore) [9]. This chromoionophore can exist in three modifications, neutral, basic and complexed by a metal ion (see Fig. 4).

Complexation constants were determined spectrophotometrically. In methanol the compound is selective for $\mathrm{Ca}^{2+}$ and $\mathrm{Ba}^{2+}$ (association constants

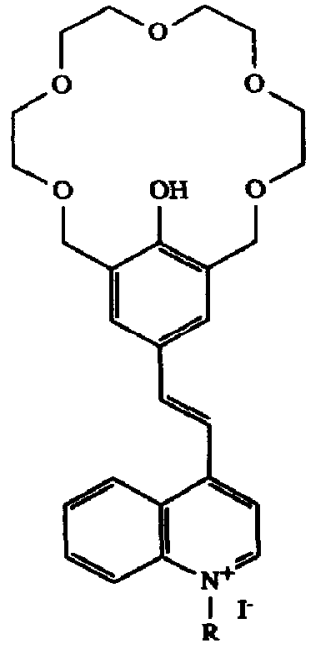

A

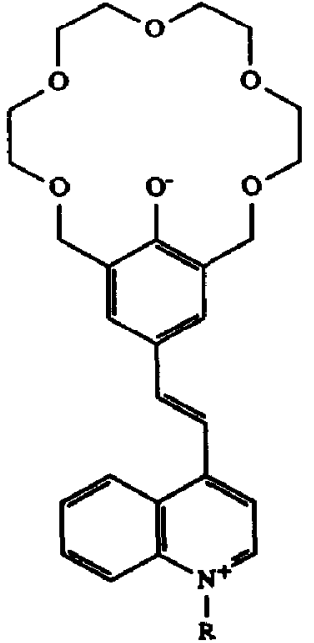

B

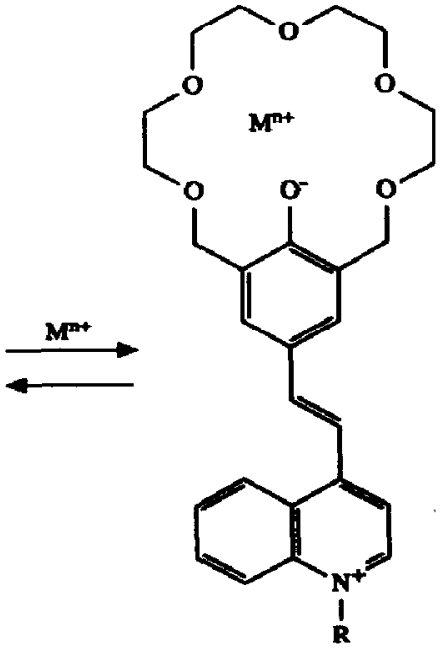

C

Fig. 4. The chromoionophore in neutral (A) and basic form (B) and its complexed form (C). $\mathrm{M}^{n+}$ represents a metal ion. 

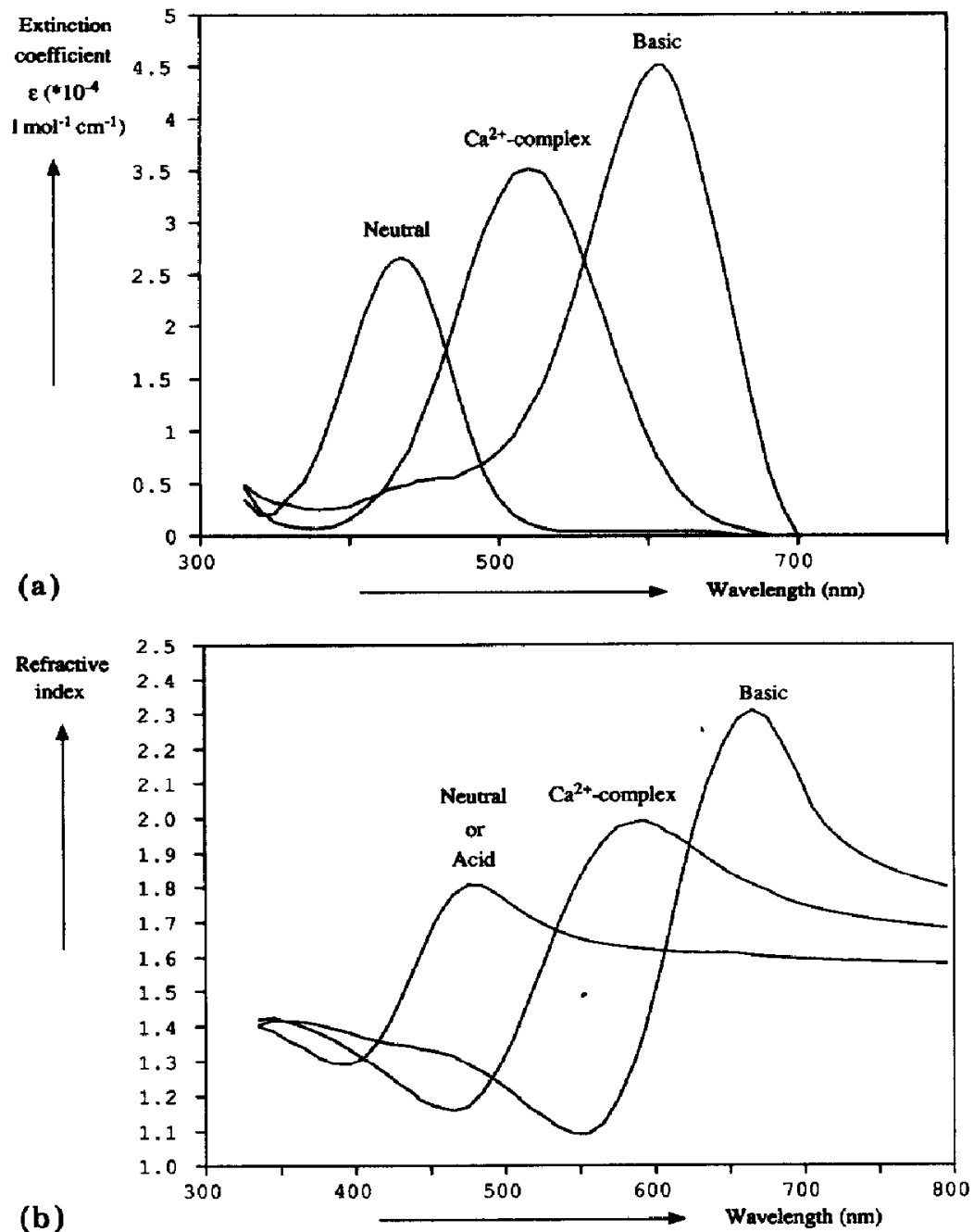

Fig. 5. (a) Absorption spectra and (b) wavelength-dependent refractive index of the chromoionophore in neutral and basic environments, and of its $\mathrm{Ca}^{2+}$ complex ( $1 \mathrm{~mol} / 1$ solution).

for both ions, $\left.1.0 \times 10^{5} \mathrm{dm}^{3} \mathrm{~mol}^{-1}\right)$. Monovalent cations $\left(\mathrm{Li}^{+}, \mathrm{Na}^{+}\right.$and $\left.\mathrm{K}^{+}\right)$ and $\mathrm{Mg}^{2+}$ have much lower association constants. The sensitivity is determined by the absolute values of the complexation constants and the selectivity by their relative values.

The absorption spectra of these chromoionophore modifications are presented in Fig. 5(a), and Fig. 5(b) shows the calculated refractive index as a function of the wavelength. It can be derived from these curves that it is suitable to use $\mathrm{He}-\mathrm{Ne}$ light $(632.8 \mathrm{~nm})$ for the measurements.

\section{Immobilization}

Three basic set-ups of the sensing layer are possible:

(1) a monolayer of the sensing material; 
(2) a thick layer of pure sensing material;

(3) the sensing material dissolved in a polymer matrix.

Figure 6 gives a simulation-based impression of the sensitivity of these three sensor set-ups. The strong and weak points of these three basic set-ups are summarized in Table 1.

We can conclude from Table 1 that a monolayer gives the best sensor performance when response time is an important factor. If response time is not important, a thicker layer would give a better sensor performance. The maximum thickness is restricted by the refractive indices of the prism and the sensor layer.

Two different lay-outs of the sensor provided with monolayers of chemo-optical interfaces have been investigated:

(1) An $\mathrm{SiO}_{2}$ surface was silylated with trimethoxysilylpropanethiol. The resulting $\mathrm{SH}$ groups on the surface were oxidized with $\mathrm{KMnO}_{4}[10]$. In this way we created a surface with $\mathrm{SO}_{3}{ }^{-}$groups. The dye was immobilized

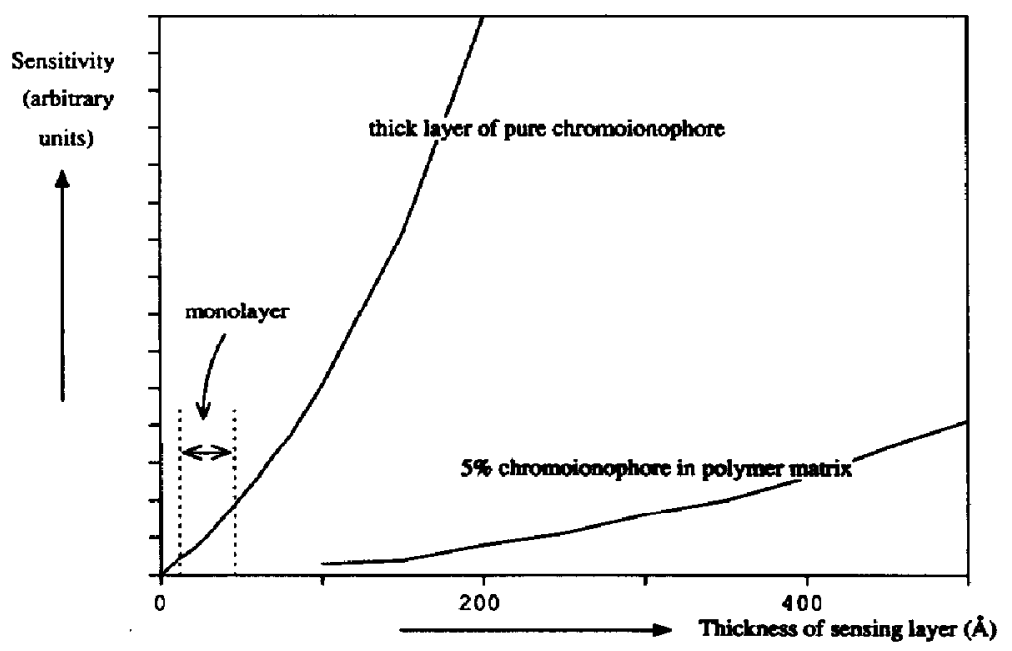

Fig. 6. Effect of immobilization method on the sensitivity of the sensor. A monolayer has a thickness of $10-50 \AA$.

TABLE 1

Advantages and disadvantages of three possible sensor set-ups

\begin{tabular}{|c|c|c|c|}
\hline Aspect & Set-up 1: monolayer & $\begin{array}{l}\text { Set-up } 2: \text { thick layer } \\
\text { of pure chromo- } \\
\text { ionophore }\end{array}$ & $\begin{array}{l}\text { Set-up 3: chromo- } \\
\text { ionophore in } \\
\text { polymer matrix }\end{array}$ \\
\hline Sensitivity & + & ++ & - \\
\hline $\begin{array}{l}\text { Response time } \\
\text { Stability }\end{array}$ & $\begin{array}{l}++ \\
+\mathbf{b}\end{array}$ & $\overline{+b}$ & $\overline{-}$ \\
\hline Ease of production & - & + & ++ \\
\hline
\end{tabular}

++ Very good, + good, - bad.

a For a comparison of the relative sensitivities, see Fig. 6.

bepends on the method of immobilization. 
by ion exchange. However, if the sensing material comes into contact with an ion-containing solution, it will dissolve by ion exchange. This method of immobilization can be applied for gas-phase sensing techniques, e.g., detection of ammonia.

(2) A chromoionophore with a long hydrocarbon chain has successfully been synthesized $\left(R=\mathrm{C}_{16} \mathrm{H}_{33}\right)$. This material can be immobilized by dipcoating on a silver surface (an intermediate layer is not necessary). In this way we were able to produce a layer $\sim 6 \mathrm{~nm}$ (equal to 1.5 monolayers) thick. Because of the hydrocarbon chain, this compound is almost completely insoluble in water, therefore these interface layers are quite stable in aqueous solutions.

The best method of immobilization is known to be covalent chemical bonding of the sensing material to the sensor surface. This is one of the topics currently under investigation.

\section{Performance of the sensor}

In the gas phase it is possible to interchange the chromoionophore between the neutral (yellow) and basic (blue) form with clean air and ammonia vapour respectively. The colour change of a $6 \mathrm{~nm}$ thin film can even be observed by direct visual inspection. Quantitative determinations can be performed by measuring the surface plasmon resonance of the system. The SP-dip shifts from $\theta=35.44^{\circ}$ (neutral) to $35.56^{\circ}$ (basic) at a wavelength of $632.8 \mathrm{~nm}$. The minimum angle difference that can be measured is $0.01^{\circ}$. This means that the maximum error of the measurement is about $9 \%$.

Our current research is concentrated on the performance of the second type of interface, which is able to measure $\mathrm{Ca}^{2+}$ concentrations in aqueous solutions.

\section{Discussion and conclusions}

We have developed a chromoionophore with good optical properties for application in an SPR-based sensor. Immobilization of this compound on the surface of the sensor appears to be possible; further research concerning this point is in progress. The dynamic range is not yet determined. It depends on the properties of the sensing material as well as on the applied sensing technique.

If the chromoionophore is used for ion sensing in an aqueous solution, the $\mathrm{pH}$ of the solution should be controlled, because the optical properties of the sensing layer depend on the $\mathrm{pH}$. The $\mathrm{p} K_{\mathrm{a}}$ of the compound is about 8 in an aqueous solution. The SPR technique in this configuration has a poor performance. Other techniques, such as direct absorption measurement with an optical fibre or a slab waveguide as well as quenching of the luminescence, will show a greater sensitivity [2]. However, the SPR technique can be fast because of the very thin sensing layers. 


\section{References}

1 C. Nylander, B. Liedberg and T. Lind, Gas detection by means of surface plasmon resonance, Sensors and Actuators, 3 (1982) $79-88$.

2 H. J. M. Kreuwel, P. V. Lambeck, J, van Gent and Th, J. A. Popma, in A. M. Scheggi (ed.), SPIE, 798 (Fiber Optic Sensors $I I, 1987$ ), pp. 218 - 224.

3 J. F. Alder, D. C. Ashworth, R. Narayanaswamy, R. E. Moss and I. O. Sutherland, An optical potassium ion sensor, Analyst, 112 (1987) 1191.

4 E. Burstein, A. Hartstein, J. Schoenwald, A. A. Maradudin, D. L. Mills and R. F. Wallis, in E. Burstein and F. de Martini (eds.), Proc. 1st Taormina Res. Conf. on the Structure of Matter, Italy, 1972, Pergamon, Oxford, 1974, pp. 89 - 108.

5 E. Kretschman, The determination of the optical constants of metals by excitation of surface plasmons, $Z$. Phys., 241 (1971) 313.

6 J. van Gent, H. J. M. Kreuwel, P. V. Lambeck, Th. J. A. Popma, G. J. Gerritsma, E. J. R. Sudhölter and D. N. Reinhoudt, to be published.

7 R. Loudon, The Quantum Theory of Light, Clarendon Press, Oxford, 1981, p. 64 onwards.

8 H. J. M. Kreuwel, Planar waveguide sensors for the chemical domain, Thesis, University of Twente, 1988, p. 52.

9 J. van Gent, E. J. R. Sudhölter, P. V. Lambeck, Th. J. A. Popma, G. J. Gerritsma and D. N. Reinhoudt, A chromogenic crown ether as a sensing molecule in optical sensors for the detection of hard metal ions, J. Chem. Soc. Chem. Commun., in press.

10 S. Suzuki, Y. Ono, S. Nakata and S. Asaoka, Structures and thermal and hydrothermal stabilities of sulfonated poly(organosiloxanes) by ${ }^{29} \mathrm{Si}$ and ${ }^{13} \mathrm{C} \mathrm{CP} / \mathrm{MAS} \mathrm{NMR}, J$. Phys. Chem., 91 (1987) $1659-1663$. 